\title{
Tensor Glyph Warping - Visualizing Metric Tensor Fields using Riemannian Exponential Maps
}

\author{
Anders Brun ${ }^{1,2}$ and Hans Knutsson ${ }^{1,2}$ \\ 1 Department of Biomedical Engineering, Linköping University, Sweden \\ 2 Center for Medical Image Science and Visualization (CMIV), Linköping, Sweden \\ $\{$ andbr, knutte\}@imt.liu.se
}

Summary. The Riemannian exponential map, and its inverse the Riemannian logarithm map, can be used to visualize metric tensor fields. In this chapter we first derive the well-known metric sphere glyph from the geodesic equations, where the tensor field to be visualized is regarded as the metric of a manifold. These glyphs capture the appearance of the tensors relative to the coordinate system of the human observer. We then introduce two new concepts for metric tensor field visualization: geodesic spheres and geodesically warped glyphs. These additions make it possible not only to visualize tensor anisotropy, but also the curvature and change in tensorshape in a local neighborhood. The framework is based on the $\exp _{p}\left(v^{i}\right)$ and $\log _{p}(q)$ maps, which can be computed by solving a second order Ordinary Differential Equation (ODE) or by manipulating the geodesic distance function. The latter can be found by solving the eikonal equation, a non-linear Partial Differential Equation (PDE), or it can be derived analytically for some manifolds. To avoid heavy calculations, we also include first and second order Taylor approximations to exp and log. In our experiments, these are shown to be sufficiently accurate to produce glyphs that visually characterize anisotropy, curvature and shape-derivatives in smooth tensor fields.

\section{Introduction}

The need for tensor visualization has grown over the past twenty years along with the advancement of image analysis, computer graphics and visualization techniques. From being an abstract mathematical entity known mostly by experts in continuum mechanics and general relativity, tensors are now widely used and visualized in applied fields such as image analysis and geology. In particular, there has been an expansion over the years, from using tensors mostly in mathematical theories of the world, towards estimating tensor quantities from experimental data. See for instance [25] for a recent survey of both techniques and applications.

We propose a technique to paint tensor glyphs in a special warped coordinate system, to enhance the visualization of curvature and partial derivatives of a metric tensor field. Glyphs are commonly used to represent the state of a tensor field 
pointwise and their collective behavior, when e.g. arranged in a grid, help to perceptualize the change of shape and orientation of the tensors. Inspired by others who have visualized tensor fields by regarding the tensor fields as the metric of a manifold, see for instance $[24,14,18,10,7,15]$, we propose a method to deform the glyphs in accordance with the metric.

One of the most exciting areas where tensor data is derived from experiments is the medical imaging modality called Diffusion Tensor MRI (DT-MRI). It is now becoming so central that clinical radiologists in general need to understand and visualize tensor fields representing in vivo water diffusion in the human brain. Fortunately, the positive definite matrices found in DT-MRI data can be visualized using ellipses (2-D) or ellipsoids (3-D), making the data understandable without knowing the details of tensor algebra. In DT-MRI, the ellipsoids are elongated along the directions of maximum water diffusion and it turns out that the shape of them are interpretable as anatomical properties of the tissue being studied. In the human brain for instance, they are elongated in the directions of nerve fiber bundles in white matter, because water diffusion is restricted in the directions perpendicular to the fibers. In the ventricles on the other hand, where the water molecules in the cerebrospinal fluid (CSF) diffuse freely in all three directions, the ellipsoids are large and spherical. These properties of ellipsoid glyphs make DT-MRI datasets easier to comprehend for a medical expert.

Tensors are mathematical objects with special geometrical properties. Most of the research in tensor visualization has focused on the most commonly used low order tensors, in particular vectors (first order, 1-D arrays) and matrices (second order, 2-D arrays). In this chapter, we study the visualization of metric tensor fields in $\mathbb{R}^{n}$, where each tensor is second order tensor. These can be represented by $n \times n$ matrices, elements of $\mathbb{R}^{n} \otimes \mathbb{R}^{n}$, which are symmetric and positive definite, i.e. they have positive eigenvalues. We call these tensor fields metric tensor fields, since they may be interpreted as the metric of a Riemannian manifold. The pointwise inverse of a DT-MRI tensor field can be regarded as a metric tensor field [18]. Structure tensor fields in image analysis, see e.g. [9], is another example of metric tensor fields.

\section{Glyphs and Glyph Warping}

A tensor glyph is a geometric object that graphically represents the local characteristics of the tensor field in a point. If a pre-filtering is applied to the field, in which the field is averaged over a small neighborhood to avoid aliasing effects or noise, one can argue that the glyph actually represents the average characteristics of the tensor field over a small area. The topic of this chapter is "glyph warping", which refers to a process of deforming glyphs according to the local characteristics of a tensor field or manifold. By construction, most kinds of tensor glyphs are scaled according to the tensor in a point, regarding this tensor as the local metric. The eigenvalues and eigenvectors of the tensor determines the stretching and rotation of the different axes of the template that is stretched to form the glyph or determine the shape of the glyph in other ways if the glyphs are constructed by a more involved process. Some glyphs based on a template are symmetric, ellipses (2-D) and ellipsoid (3-D) glyphs can be constructed from a circle or a sphere. Other glyphs, such as the "space-ship glyphs" found in [27] or the superquadric tensor glyphs [13], are not rotationally symmetric but has three or more symmetry axes. The curvature 
and the partial derivatives of the metric tensor field are usually not represented by individual glyphs. It can usually be inferred from the collective appearance of the glyphs in a neighborhood, i.e. if the glyphs tend to rotate or change in size. This effect could also be visualized by warping or deforming the glyphs, i.e. bending them like a banana or let them change size. If we regard the tensor field as the metric of a manifold, i.e. a curved geometric space, one natural way to think of this deformation process is to imagine that the glyph is placed inside the manifold. However, while the glyph template is defined in a vector space, the manifold is curved and there is in general no way to fit the flat template inside the manifold while at the same time preserving distances in the glyph if we compare Euclidean distances in the glyph template with geodesic distances in the manifold. The situation is very much the same in map making, i.e. there is no way to map the surface of the Earth to a flat vector space, so that all geodesic distances are mapped to Euclidean distances in the new space.

Using the exp and log maps to deform the glyph, which is what is described in this chapter, is a compromise and this mapping will at least preserve radial distances and radial angles in the glyph.

\section{Related Work}

In 1881 the French cartographer Nicolas Auguste Tissot published ideas on using circles and ellipses to visualize the deformation of map projections. Mapping the Earth to a flat surface is not possible without introducing some kind of angular or area distortion in the process. The Tissot indicatrix, see Fig. 3, is a small circle or ellipse painted in a map projection. It represents the deformation of an infinitely small circle on the Earth after being deformed by the map projection. If the Tissot indicatrix is a perfect circle, and not an ellipse, then the projection is angle preserving (conformal), and if the area of Tissot indicatrices does not change across the map projection, the map projection is area preserving (authalic). A natural extension of the Tissot indicatrix is to use geodesic distances on the Earth (ellipsoid) to define the circle, in general resulting in a distorted ellipse. For this reason the geodesic sphere glyph we propose in this chapter, for the visualization of arbitrary metric tensor fields, can be seen as a generalization of the original Tissot indicatrix. In Fig. 2 and 2 we show how the geodesic variant of the Tissot indicatrix may be used to visualize the deformation of the metric in a projection of two mathematical surfaces, a half-sphere and a cone.

Later work in computer graphics has also described methods to visualize the distortion of a projected surface, or manifold in general, from the information contained in a metric tensor field. In spot noise [24], a small image or spot, is pasted stochastically in multiple copies over a parametric surface to create different textures. The original paper on spot noise also demonstrates how anisotropic spot noise, in the 2-D texture coordinate system of a curved surface embedded in $3-\mathrm{D}$, results in isotropic patterns in object space. This is in fact a way to visualize the metric tensor of the surface. Textures have also been used to visualize vector fields. In line integral convolution (LIC) [5], vector fields are visualized by convolution (integration) of a random texture with streamlines created from the vector field. This yields a low-frequency response along the streamlines. In a method similar to spot noise and 

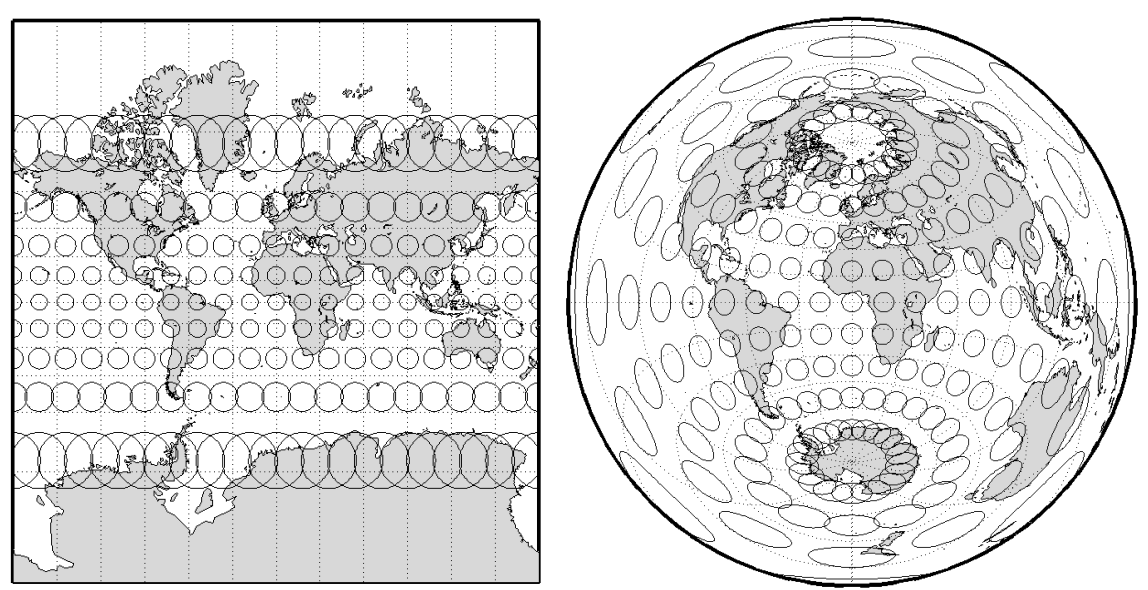

Fig. 1. The area and angle distortion of map projections visualized using Tissot indicatrices. Left: The Mercator projection, used in e.g. Google Maps. It is conformal. Right: The Equidistant Azimuthal projection. It is neither conformal nor authalic.

LIC, noise is filtered by anisotropic filters steered by second order tensors to visualize the tensor field, see for instance [16] for an early example or [22, 17]. Another example of second order tensor field visualization include the Hyper-LIC [29], an extension of the LIC method where the convolution proceeds not only along a single streamline, but along a non-linear patch which is aligned with streamlines derived from both the first and second eigenvectors of the tensors. This is somewhat similar to the approach taken in this chapter, since a warped coordinate system is created which can be used for glyph warping. In [10] an approach is presented based on a physical interpretation of the tensor field and it is also able to, in contrast to many other methods, visualize second order tensors with negative eigenvalues. Finally a procedural generation of textures from tensor fields have been investigated in [14], where reaction-diffusion patterns are steered by the metric tensor field. This yields a pattern that seems to be composed by separate glyphs, ellipses in 2-D, which are adaptively placed, scaled and deformed by the tensor field. For a successful implementation of this method, one has to overcome the numerical problems of simulating a highly non-linear PDE.

In the medical community, there has been a special need to extract information from tensor fields that goes beyond the visualization of local properties of the field. In "tractography", entire tracts are visualized by performing streamline tracking along the main eigenvector field of a second order tensor field. This procedure, called "fiber tracking", helps radiologists to locate fiber bundles in the human brain and find out about long range white matter fiber connectivity. Fiber tracking shares many similarities with the LIC, Hyper-LIC and Hyper-streamlines [6], but it is also a research topic in its own right since it is heavily biased by clinical needs and the quest for anatomical understanding of the human brain.

Two properties of spot noise and reaction-diffusion visualization seem to be important for the quality and perception of the tensor visualization. First, both of these methods spread the glyph-like spots in a uniform way according to the tensor 

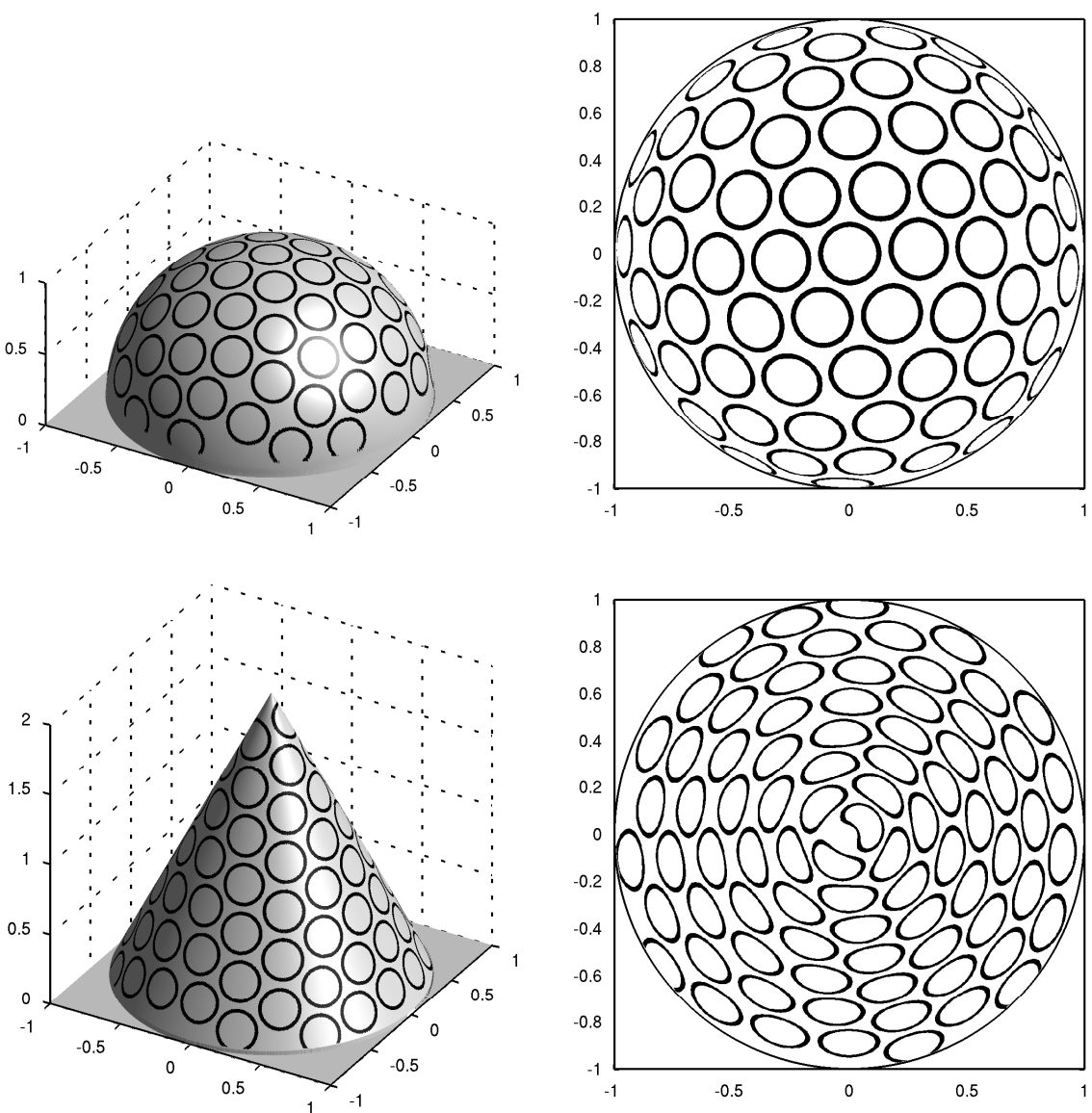

Fig. 2. Left: A half sphere in $\mathbb{R}^{3}$ painted with geodesic spheres. Right: A 2-D chart describing the half-sphere, i.e. the z-direction has been removed. The same geodesic spheres efficiently visualize the space-variant metric. Left: A cone sphere in $\mathbb{R}^{3}$ painted with geodesic spheres. Right: A 2-D chart describing the cone, i.e. the z-direction has been removed. The same geodesic spheres efficiently visualize the space-variant metric. Note in particular the banana-shaped spheres in the center and the more ellipse-shaped spheres close to the perimeter.

field regarded as a metric. The latter of these methods not only scale but also bend the glyph-like structures according to the curvature of the tensor field. In recent work on glyph packing [15] and anisotropic noise sampling [7], the first of these behaviors is mimicked and glyphs are placed uniformly over the field. However, the glyphs themselves are still based on the value of the tensor field in each point and do not consider curvature. In this chapter, we present glyphs that do exactly that: they bend, expand and contract according to the derivative of the tensor field. In combination with a glyph-packing procedure, this technique has the potential to 
mimick the two most desirable properties of the reaction-diffusion, in a framework that is numerically stable and fast to compute.

The work presented here is also related to work on texture mapping in computer graphics, in particular the decal compositing with discrete exponential maps [20]. Decal compositing refers to the mapping of small texture maps, decals, onto surface models embedded in $\mathbb{R}^{3}$. It has been used mainly for artistic purposes and it is defined only for 2-D surfaces embedded in 3-D. Other methods for the calculation of exponential maps on general manifolds have also been presented. In [21] fast marching is presented as a means to calculate geodesics emanating from a point, i.e. indirectly the calculation of exponential maps. In [28] fast methods are presented to calculate all geodesics in a manifold, starting from any point in any direction and traveling any distance. Finally in [4] and [3], the LogMap method is presented as a means of calculating the inverse of the Riemannian exponential map, a method which is reviewed later in this chapter.

\section{Tensors and Index Notation}

Tensors generalize scalars, vectors and matrices to higher dimensions. Sometimes the word "tensor" is used for any multi-dimensional array with more indices than a matrix, i.e. more than two. We use the term in a more precise manner that is in agreement with the notation in physics and differential geometry. In these fields of research, tensors are geometric objects that are invariant under coordinate changes. A vector is a tensor, i.e. it is a geometric object that remains the same regardless of the choice of basis or coordinate system that is used to descibe it. In physics the word "tensor" usually refers to what in mathematics would be called a "tensor field" but in both domains it is meaningful to think of tensors as objects defined pointwise in a vector space $V$.

Many spatial quantities in physics are tensors, for instance; velocity $(\mathrm{m} / \mathrm{s})$, diffusion $\left(\mathrm{m}^{2} / \mathrm{s}\right)$ and electric field strength $(\mathrm{V} / \mathrm{m})$. In mathematics, contravariant vectors are those that transform like velocity and position vectors, while the so called covariant vectors transform like gradients under a change of coordinate system. Examples of higher order tensors in mathematics are for instance quadratic forms. For a general definition, a tensor $F$ is defined as multi-linear map,

$$
F: \underbrace{V^{*} \times \ldots \times V^{*}}_{r} \times \underbrace{V \times \ldots \times V}_{s} \rightarrow \mathbb{R}
$$

i.e. a map that is linear in each of its arguments. Its order is $r+s$ and it has type $(r, s)$, meaning that it operates on $r$ covariant vectors and $s$ contravariant vectors. In some contexts, order is called rank and type is called valence, which can be confusing since rank is also used to describe the rank of matrices. Similar to vectors and the metric previously defined, the action of tensors can be defined by components that are derived from the action on all combinations of basis vectors $\left\{\mathbf{w}^{i}\right\}$ in $V^{*}$ and $\left\{\mathbf{b}_{j}\right\}$ in $V$,

$$
F_{j_{1}, j_{2}, \ldots, j_{s}}^{i_{1}, i_{2}, \ldots, i_{r}}=T\left(\mathbf{w}^{i_{1}}, \ldots, \mathbf{w}^{i_{r}}, \mathbf{b}^{i_{1}}, \ldots, \mathbf{b}^{i_{r}}\right) .
$$

The number of components is $n^{r+s}$. If the coordinates are changed, $\tilde{x}^{i}=t_{k}{ }^{i} x^{k}$, then each contravariant index is transformed as a vector and each covariant index is transformed as a dual vector, 


$$
\tilde{F}_{x y z}^{a b c}=F_{x y z}^{a b c} t_{a}{ }^{i} t_{b}{ }^{j} t_{c}{ }^{k} \ldots\left(t^{-1}\right)^{x}{ }_{m}\left(t^{-1}\right)^{y}{ }_{n}\left(t^{-1}\right)^{z}{ }_{o} \ldots
$$

In physics, this is sometimes how tensors are defined, i.e. as objects that transform according to certain transformation laws.

From here on, we will use index notation, which is commonly used in differential geometry to denote tensors and differentiate between covariant (lowered) and contravariant (raised) indices. For an introduction to tensors and tensor notation, see for instance $[11,26]$. In order to make the interpretation accessible to a broader audience, we will not use the customary Einstein summation convention, meaning that all sums will be written out explicitly instead. In index notation a (contravariant) vector is identified with its coordinates, meaning that a vector $\mathbf{v}$ in Euclidean space $\mathbb{R}^{n}$ is written using its coordinates $v^{i}$ in some basis,

$$
\mathbf{v}=v^{i}=\sum_{i=1}^{n} v^{i} \mathbf{b}_{i}
$$

Note in particular that the basis vectors have been dropped and are assumed implicitly in the short form $v^{i}$. The index variable $i$ is an integer in the range $1 \ldots n$ and it is type set in superscript to indicate that this index, and this vector, is contravariant. To further increase readability we will also write equations in ordinary linear algebra notation when possible, i.e. bold face lower case letters for both contravariant and covariant vectors $(\mathbf{v}, \mathbf{x}, \ldots)$ and upper case bold letters for matrices $(\mathbf{A}, \mathbf{G}, \ldots)$. In some expressions we use $\dot{x}^{i}$ and $\ddot{x}^{i}$ to denote first- and second order time derivatives.

In addition to vectors, we will consider higher order tensors in this chapter, in particular the metric tensor. The metric tensor is a mathematical object which defines the scalar product between (contravariant) vectors, which in turn can be used to measure important properties in space such as lengths, angles, area and so on. In vector algebra the scalar product is often implicitly defined simply by

$$
\langle\mathbf{v}, \mathbf{u}\rangle=\mathbf{v}^{T} \mathbf{u}=\sum_{i=1}^{n} v^{i} u^{i}
$$

but in general any symmetric positive definite $n \times n$-matrix $\mathbf{G}$ can be used to define a metric,

$$
\langle\mathbf{v}, \mathbf{u}\rangle_{G}=\mathbf{v}^{T} \mathbf{G u}=\sum_{i=1}^{n} \sum_{j=1}^{n} v^{i} g_{i j} u^{j} .
$$

The latter also introduces the commonly used tensor notation for the metric, i.e. lowercase with indices written in subscript $g_{i j}$. In index notation, upper- and lower case letters have less meaning and to comply with standard notation in both linear algebra and differential geometry, we will denote the metric by either $g_{i j}$ or $\mathbf{G}$. Subscript indices indicate that the metric is a covariant tensor. In tensor algebra it is natural to pair contravariant indices with covariant ditto, so the previous expression in Eq. 5 for a scalar product is somewhat odd. Instead, it is better to write out the metric explicitly,

$$
\langle\mathbf{v}, \mathbf{u}\rangle=\mathbf{v}^{T} \mathbf{u}=\sum_{i=1}^{n} v^{i} \delta_{i j} u^{j},
$$

where $\delta_{i j}$ is the Kronecker delta symbol, defined by $\delta_{i j}=1$ for $i=j$ and 0 elsewhere. It can be regarded as the unit-metric. Now the number of contravariant (upper) and 
covariant (lower) indices match, meaning that the result of the calculation is a scalar (no index).

In summary, the index notation is a handy way to denote vectors and matrices, which easily extends to higher dimensions by adding more indices. At a first glance, the common notation for vectors and matrices may seem more intuitive, but three things are easier to do in index notation. First, index notation extends naturally to higher order tensors, i.e. objects with three or more indices. Secondly, index notation can differentiate between covariance and contravariance by the use of upper- and lower indices. It should also be noted that index notation is particularly useful when used in combination with the Einstein summation convention, meaning that the summation symbol $\sum_{i=1}^{n}$ is omitted from all expressions and instead it is assumed that indices $i, j$ etc appearing more than one time in an expression is summed over, from $1 \ldots n$. In this notation the above scalar product is simply

$$
\langle\mathbf{v}, \mathbf{u}\rangle_{g}=v^{i} g_{i j} u^{j}=g_{i j} v^{i} u^{j}=g_{i j} u^{j} v^{i} .
$$

From the example it is also easy to see another advantage with the index notation, namely that the ordering of the tensors is irrelevant, in contrast to matrix and vector notation.

\section{The Metric and Metric Spheres}

We will now take a closer look at the metric, or metric tensor, and see how we can visualize a metric. We will also introduce a particular orthonormal $(\mathrm{ON})$ coordinate system that will be useful later in the chapter.

The metric is the object specifying the scalar product in a particular point on a manifold in differential geometry. It encodes how to measure lengths, angles and area in a particular point on the manifold by specifying the scalar product between tangent vectors in this particular point. A natural way to visualize the metric is to visualize a "unit sphere", i.e. a sphere with radius equal to 1. By "natural" we do not necessarily mean the most suitable way to visualize a metric from a human perception point of view, but rather a straightforward way to visualize the metric using simple mathematics. In Euclidean space the unit sphere is the set of points, $\mathbf{x} \in \mathbb{R}^{n}$, satisfying $\|\mathbf{x}\|=\sqrt{\langle\mathbf{x}, \mathbf{x}\rangle}=1$. In tensor notation and with an arbitrary metric $g_{i j}$ this translates to

$$
\sum_{i=1}^{n} \sum_{j=1}^{n} g_{i j} x^{i} x^{j}=1 .
$$

While the metric $g_{i j}=\mathbf{G}$ may be interpreted as a symmetric positive definite matrix, it can be spectrally decomposed,

$$
\mathbf{G}=\mathbf{U} \boldsymbol{\Lambda} \mathbf{U}^{*},
$$

where $\mathbf{U}$ is a unitary matrix, $\mathbf{U U}^{*}=\mathbf{I}$, and $\boldsymbol{\Lambda}$ is a diagonal matrix with the eigenvalues of $\mathbf{G}$ ordered in descending order, $\boldsymbol{\Lambda}_{i i}=\lambda_{i}$. The eigenvectors to $\mathbf{G}$, found in the columns of $\mathbf{U}$, form an ON basis in $\mathbb{R}^{n}$ for both the standard metric $\delta_{i j}$ and in the arbitrary metric $g_{i j}$. For instance, in $\mathbb{R}^{2}$ the first eigenvector, corresponding to the eigenvalue $\lambda_{1}$, point along the major axis and the last eigenvector, corresponding 
to $\lambda_{2}$, point along the minor axis of the ellipse-shaped geodesic ball. In the general case, $\mathbb{R}^{n}$, the metric sphere will be a hyper-ellipsoid. Using this knowledge we may design a special coordinate system, which is aligned with the axes of the hyperellipsoid. If $\mathbf{U}=\left(\mathbf{e}_{1}, \mathbf{e}_{2}, \ldots, \mathbf{e}_{n}\right)$ and coordinates are denoted by $c^{i}$, a vector $\mathbf{v} \in \mathbb{R}^{n}$ is decomposed by

$$
\mathbf{v}=v^{i}=\frac{1}{\sqrt{\lambda_{1}}} \mathbf{e}_{1} c^{1}+\frac{1}{\sqrt{\lambda_{2}}} \mathbf{e}_{2} c^{2}+\ldots+\frac{1}{\sqrt{\lambda_{n}}} \mathbf{e}_{n} c^{n} .
$$

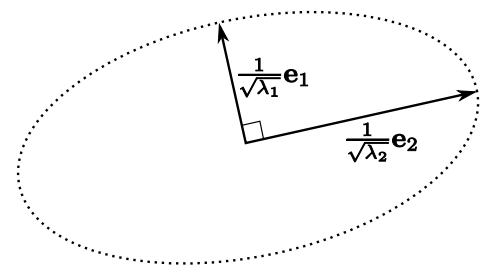

Fig. 3. Coordinate basis vectors in $\mathbb{R}^{2}$ derived for some metric $g_{i j}$. This coordinate basis is $\mathrm{ON}$ in $g_{i j}$.

This coordinate system has many advantages, in $\mathbb{R}^{2}$ for instance we may now easily parameterize the surface of the metric sphere by painting an isotropic sphere in the $c^{i}$ coordinates, $c^{1}=\cos (t)$ and $c^{2}=\sin (t), 0 \leq t<2 \pi$. An alternative approach to visualize the metric, and emphasize the direction on the eigenvectors, is to paint a unit box, $c^{i}: \max \left(c^{1}, c^{2}\right)=1$. In fact, we may paint any tensor glyph in this coordinate system, for instance superquadratic tensor glyphs [13] or even the "space ship" glyph in [27].

We call the map from this coordinate system to the vector space $E, E: \mathbb{R}^{n} \rightarrow V$. It is an isomorphism from the Euclidean space $\mathbb{R}^{n}$ (and the unit metric) to a new vector space $V$ equipped with the metric $\mathbf{G}=g_{i j}$. Of many such isomorphisms, it has the special property that it is aligned with the axes of the hyper ellipsoid describing $g_{i j}$ in $\mathrm{V}$, in a particular basis.

\section{The Geodesic Equation and Geodesic Spheres}

In applications where metric tensor fields are visualized, the metric is not constant but changes from point to point. A natural theory for space-variant metrics is the non-Euclidean geometry found in Riemannian manifolds, which has already been pointed out by a number of authors, see for instance [18]. In Riemannian geometry the distance between two points in space is defined by the length of the shortest curve between them, where the length of this curve is obtained from the integral over the tangent vectors to a curve, measured using a space-variant metric $g_{i j}(x)$,

$$
d(a, b)=\min _{\gamma: \gamma(0)=a, \gamma(1)=b} \int_{0}^{1} \sqrt{\dot{\gamma}(t)^{i} g_{i j}(\gamma(t)) \dot{\gamma}(t)^{j}} d t .
$$


Similar to the case of a constant metric, we may now define geodesic spheres in this Riemannian manifold. For a sphere centered in a point $p$ in the manifold, the following relation hold for points $x$ in the geodesic sphere,

$$
d(p, x)=1
$$

The problem with this metric, from an application point of view, is that the spacevariant metric makes it more difficult to evaluate the distance between two different points since the minimization is performed over an infinite set of curves $\gamma$.

One way to approach this problem is to derive a parametric function for points on the sphere, without measuring distances explicitly. Using the geodesic equation, defined in Eq. 14 below, geodesics emanating from a point $p$ starting off in a specific direction and traveling a specific distance (in this case 1) may be generated. These solutions correspond to paths of free particles moving in the manifold, without any forces acting on them, and in this sense they generalize the notion of straight lines in Euclidean geometry. Without going into details, geodesics can be described and calculated using the geodesic equation. It is a second order ODE which expresses that the second derivative of the position, the acceleration, is zero. Because of the space variant metric, a special term involving the Christoffel symbol needs to be included,

$$
\frac{d^{2} x^{i}}{d t^{2}}+\sum_{j=1}^{n} \sum_{k=1}^{n} \Gamma_{j k}^{i} \frac{d x^{j}}{d t} \frac{d x^{k}}{d t}=0,
$$

where $1 \leq i, j, k \leq n . \Gamma_{j k}^{i}$ is the Christoffel symbol, which is actually a function of the coordinate system, i.e. $\Gamma_{j k}^{i}\left(x^{i}\right)$. It is not a tensor in a strict sense, it does not transform as a tensor when the coordinate system is changed, but it benefits greatly from the index notation since it has three indices. It is derived from the metric tensor,

$$
\Gamma_{j k}^{i}=\frac{1}{2} \sum_{m=1}^{n} g^{i m}\left(\frac{\partial g_{m j}}{\partial x^{k}}+\frac{\partial g_{m k}}{\partial x^{j}}-\frac{\partial g_{j k}}{\partial x^{m}}\right),
$$

where $g^{i j}$ is the inverse of the metric $g_{i j}$, i.e. $g^{i j}=\mathbf{G}^{-1}$. A geodesic starting at $\gamma(0)=p$, where $p$ is a point on the manifold, with a velocity $\dot{\gamma}(0)=v^{i}$ will have a geodesic length $\left\|v^{i}\right\|=\sqrt{\sum_{i, j} v^{i} g_{i j} v^{j}}$ at $t=1$ and thus $d(p, \gamma(1))=\left\|v^{i}\right\|$. In this way, by following geodesics starting at $p$ with different unit speed tangent vectors, we obtain a polar representation of a geodesic sphere. We will return to how this is solved in practice in a later section dealing specifically with the implementation of this.

\section{The Exponential Map and Riemannian Normal Coordinates}

With the introduction of geodesic distance and geodesics, we now have a way to paint geodesic spheres to visualize some of the characteristics of a space-variant metric tensor field. However, we have not yet introduced a coordinate system similar to the coordinates $c^{i}$ introduced for a constant metric. A first step towards the introduction of such a coordinate system is to define the Riemannian exponential map, known 
from differential geometry. This map, and its inverse the Riemannian logarithm, is depicted in Fig. 4.

Let $T_{p} M$ denote the tangent space to a manifold $M$ at a point $p \in M$. In the case of our space-variant metric, this is simply the space of all tangent vectors of curves through a point $p$, which is a vector space. In particular, this is the space of all possible tangent vectors to geodesics emanating from $p$. The $\operatorname{map}_{\exp _{p}}: T_{p} M \rightarrow M$ is defined by

$$
\exp _{p}\left(v^{i}\right)=\gamma(1)
$$

where $\gamma$ is the geodesic for which $\gamma(0)=p$ and $\dot{\gamma}(0)=v^{i}$. It is appropriate to use a 'shooting' analogy here, $\exp _{p}\left(v^{i}\right)$ is where a particle ends up after one time unit, if it is shot from a point $p$ with velocity $v^{i}$.

The introduction of the exponential map can be done without any reference to coordinates in a specific basis, it is simply a map from vectors $v^{i}$ seen as geometric objects in the tangent vector space of a point $p, T_{p} M$, to other points in the manifold. By choosing an ON coordinate system for $T_{p} M$, we obtain what is called Riemannian Normal Coordinates, Geodesic Normal Coordinates or Normal Coordinates for short. This ON basis can be seen as an isomorphism $E: \mathbb{R}^{n} \rightarrow T_{p} M$. Joining it with the exponential map, we have a map from $\mathbb{R}^{n} \rightarrow M$, and the inverse of this map gives us the coordinate of a point $q$ on the manifold by $\varphi(q)=E^{-1} \exp _{p}^{-1}(q)$, which is a well defined inverse in a neighborhood $U$ around $p$. We will soon take a closer look at the inverse of the exponential map and call it $\log _{p}$.

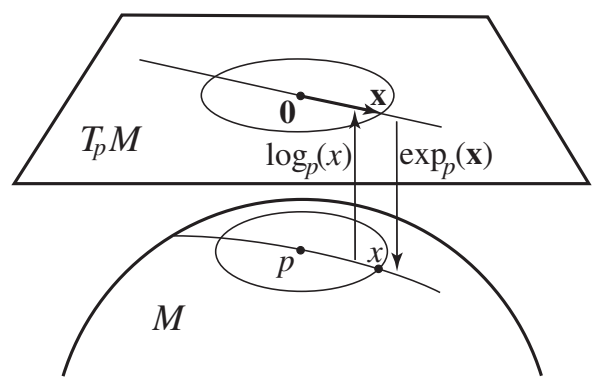

Fig. 4. A schematic view of the $\exp _{p}$ and $\log _{p}$.

\section{Solving the Geodesic Equation}

Before we actually use the geodesic equation to paint glyphs, we will briefly touch upon how to solve it, both accurately using ODE solvers and approximately using a Taylor approximation. Like any second- or higher order ODE, it can be reformulated by a system of first order ODEs, $\frac{\partial s}{\partial t}=f(s, t)$, for a vector valued state $s$. The two variables $x^{i}$ and $\dot{x}^{i}$ evolve in time according to

$$
\left[\begin{array}{c}
\frac{\partial x^{i}}{\partial t} \\
\frac{\partial \dot{x}^{i}}{\partial t}
\end{array}\right]=\left[\begin{array}{c}
\dot{x}^{i} \\
-\sum_{j=1}^{n} \sum_{k=1}^{n} \Gamma_{j k}^{i} \dot{x}^{j} \dot{x}^{k}
\end{array}\right],
$$


where the $\Gamma_{j k}^{i}$ is spatially varying depending on $x^{i}$. The right hand side is independent of $t$, i.e. it is a so called autonomous ODE.

$$
\begin{aligned}
& \frac{\partial x^{i}}{\partial t}=\dot{x}^{i} \\
& \frac{\partial \dot{x}^{i}}{\partial t}=-\sum_{j=1}^{n} \sum_{k=1}^{n} \Gamma_{j k}^{i} \dot{x}^{j} \dot{x}^{k} .
\end{aligned}
$$

Given that initial conditions are known, e.g. $x(0)=p$ and $\dot{x}(0)=v^{i}$, this system of ODEs has exactly one unique solution under very general conditions according to the Picard-Lindelöf theorem. We identify a particular solution with a geodesic curve $x^{i}(t)=\gamma(t)$. While the Christoffel symbol might be difficult to comprehend at first, it is worth noting that the contribution by $\Gamma_{j k}^{i}$ is symmetric with respect to a flip of sign in $\dot{x}^{i}$. Implementation of a numerical solution to this ODE in e.g. Matlab is straightforward using standard ODE solvers. The only reservation is that even a third order tensor-like object, like the Christoffel symbol, generates a notation which is quite involved when implemented in a vector- and matrix oriented language like Matlab. It is also important to use a proper interpolation scheme in the calculation of derivatives of $g_{i j}$, if the tensor field is known only from samples. We used cubic interpolation. To ensure positive definiteness we performed the interpolation in the Log-Euclidean domain [1]. It is however important to point out that the proper choice of interpolation method highly depends on the application, and the glyph warping framework we present is applied after the user has decided upon the right choice of interpolation. In some applications the continuous tensor field will be known in every point, while in others the user may choose a model where the tensor field is constant inside each pixel or voxel. In the latter case the solution to the geodesic equation must be generalized to non-continous tensor fields which, in terms of optics and light-beams that are actually geodesics, corresponds to a generalized law of refraction on the boundary between two neighboring pixels or voxels.

For many applications in computer graphics, speed and ease of implementation is an issue. For this reason we will also derive Taylor approximations of the exponential map. Directly from the geodesic equation, we have the second order derivative of our geodesic curve. Given the initial value of the position and derivative, $x(0)$ and $\dot{x}(0)$, we have everything needed in order to make a second order Taylor approximation of a geodesic, valid for small values of $t$ :

$$
\tilde{x}^{i}(t)=x^{i}(0)+\dot{x}^{i}(0) t-\frac{1}{2} \sum_{j=1}^{n} \sum_{k=1}^{n} \Gamma_{j k}^{i} \dot{x}(0)^{j} \dot{x}(0)^{k} t^{2}+O\left(\left\|\dot{x}(0)^{i} t\right\|^{3}\right),
$$

which for $t=1$, according to Eq. 16, yields for a coordinate system in which $p^{i}=0$,

$$
\exp _{p}\left(v^{i}\right)=0+v^{i}-\frac{1}{2} \sum_{j=1}^{n} \sum_{k=1}^{n} \Gamma_{j k}^{i} v^{j} v^{k}+O\left(\left\|v^{i}\right\|^{3}\right)=q^{i}
$$

This approximation will only be valid around a small neighborhood to $p$. As of today, it is not entirely clear how good this approximation is and more research is needed, to find bounds on the approximation error and perhaps also derive higher order Taylor approximations for geodesics. As will be shown in the experimental section, this approximation is however good enough to be useful. 


\section{Geodesic Spheres and Warped Coordinate Systems}

Using Eqs. 16, 17 or the approximation Eq. 21, we are able to explicitly map unit vectors in $T_{p} M$ to coordinates on the manifold and thereby paint unit spheres. By choosing the special coordinate system derived above, $c^{i}$, in combination with these formulas, we may also navigate on the manifold using a Riemannian normal coordinate system that is aligned with the major and minor axes of the unit circle. This allows us to map not only spheres, but in fact any glyph that is naturally defined in the ellipse- or ellipsoid aligned coordinate system. In this chapter we will demonstrate this by mapping the aligned unit box by using Riemannian normal coordinates. This will result in a box glyph with approximately unit length sides, which has its major axis along the main eigenvector of the local metric, but on a larger scale has its shape deformed according geodesics emanating from its center point.

\section{The Logarithmic Map}

The function $\log _{p}(q)$ is a function which maps points $q$ on the manifold to the tangent space in $p, T_{p} M$, and it is the inverse of $\exp _{p}\left(v^{i}\right)$. While the exponential function is fairly easy to calculate numerically by solving a second order ODE, the estimation of the $\log _{p}(q)$ mapping has attracted less attention in the literature, perhaps by the infeasibility of fast and accurate solutions. From the Taylor approximation in Eq. 21 it is however straightforward to derive the second order Taylor approximation for this inverse,

$$
\log _{p}\left(q^{i}\right)=0+q^{i}+\frac{1}{2} \sum_{j=1}^{n} \sum_{k=1}^{n} \Gamma_{j k}^{i} q^{j} q^{k}+O\left(\left\|q^{i}\right\|^{3}\right) .
$$

In our experience this approximation is less stable than the Taylor approximation of $\exp _{p}\left(v^{i}\right)$ in Eq. 21, i.e. it is only valid in a small neighborhood around $p$, and for this reason we have not used the second order Taylor approximation of this mapping in our experiments.

A recently proposed method to calculate the $\log _{p}(q)$ map is the LogMap method $[3,4]$. One way to explain this method is to study how the intrinsic mean is computed $[12,8]$. Let $\left\{x_{i}\right\}$ be $N$ data points in a manifold $M$ and seek the minimizer to the function

$$
f(p)=\frac{1}{2 N} \sum_{i=1}^{N} d^{2}\left(p, x_{i}\right),
$$

where $d^{2}\left(p, x_{i}\right)$ is the squared geodesic distance between points $p$ and $x_{i}$. Then the gradient of $f$ is $[19,12]$

$$
\nabla f(p)=-g_{s t} \frac{1}{N} \sum_{i=1}^{N} \log _{p} x_{i}
$$

Setting $N=1$ and $x_{1}=x$ gives the following formula for $\log _{p}$,

$$
\log _{p}(x)=-\left.g^{s t} \frac{1}{2} \nabla_{y} d^{2}(y, x)\right|_{y=p} .
$$


The metric $g_{s t}$ and the inverse metric $g^{s t}=\left(g^{-1}\right)^{s t}$ have been added here to handle the general case, but choosing an ON-basis for $T_{p} M$ yield $g_{s t}=g^{s t}=\delta_{s t}$ and allow us to identify co- and contravariant vectors. With the formula above, estimating $\log _{p}(q)$ becomes a matter of estimating geodesic distances on $M$. If distances $d(x, y)$ are known for all $x \in N(p)$, where $N(p)$ is some small neighborhood of $p$, and for all $y \in M$, then the gradient of the squared distance function can be easily estimated numerically by fitting a second order polynomial which is then differentiated analytically. (The reason for using the squared distance function is simply that it is much easier to approximate using a finite set of basis functions, compared to the plain distance function which has a discontinuity in its origin.) Distance functions in turn can be estimated numerically for manifolds by solving the eikonal equation, e.g. by using level-set methods for front propagation [18, 21], or ordered upwind methods such as fast marching [23, 21] and the Dijkstra algorithm [2]. In some special cases (e.g. the sphere, the cone, the Poisson disk model of the hyperbolic plane, ...) the distance function can also be derived analytically. In this chapter we focus mainly on the $\exp _{p}\left(v^{i}\right)$ map, since it is the most convenient mapping to use if one has a glyph that is described by a set of connected vertices. We note however that if the glyph is given by a texture, the LogMap method might be convenient since it yields a mapping from points $q$ on the manifold directly to texture coordinates $v^{i}$. It also has the computational advantage that it calculates the mapping for all points in the manifold in one step, given only a few global distance functions from points around $p$. This property makes the LogMap method more useful when many points are to be mapped, since the ODE solution of the exponential map then requires that a large set of separate ODEs are solved.

\section{Experiments}

In this section we describe some experiments performed on a simulated synthetic 2-dimensional DT-MRI dataset, where Rician noise and partial volume effects have been introduced using realistic parameter settings representative for common clinical protocols. This dataset consists of a 2 -D tensor field with $2 \times 2$ symmetric positive definite tensors. We have chosen a 2 -D dataset because it demonstrates several features of glyph warping and yet it is easy to visualize in print, but it is important to note that glyph warping using exponential maps is not restricted to 2-D, but works in any dimensions. In Fig. 5 we show a close up of the tensor field displayed using three variants of sphere-glyphs. The first variant is the metric sphere, which may be seen as a first order approximation to the geodesic equations. The second and third image shows the second order approximation and the numerically derived solution to the geodesic ODE. In Fig. 6 we demonstrate the effect on a global scale, once again we use the sphere-glyph. The difference is more subtle now, but experts in tensor image processing still agree that the two rightmost images have a softer appearance. In a third experiment, see Fig. 7, we once again tried the three variants of glyph warping, but this time we used the box glyph instead. Here the differences are more obvious. We note that both curvature and changes in tensor shape may be seen in the two rightmost visualizations. Again there is little difference between the second order Taylor approximation and the numerical ODE solution. Compared to the sphere-glyph, the box contains straight lines, which is the main reason why it is easier to see the effect of the non-linear mapping. In a fourth experiment, see 


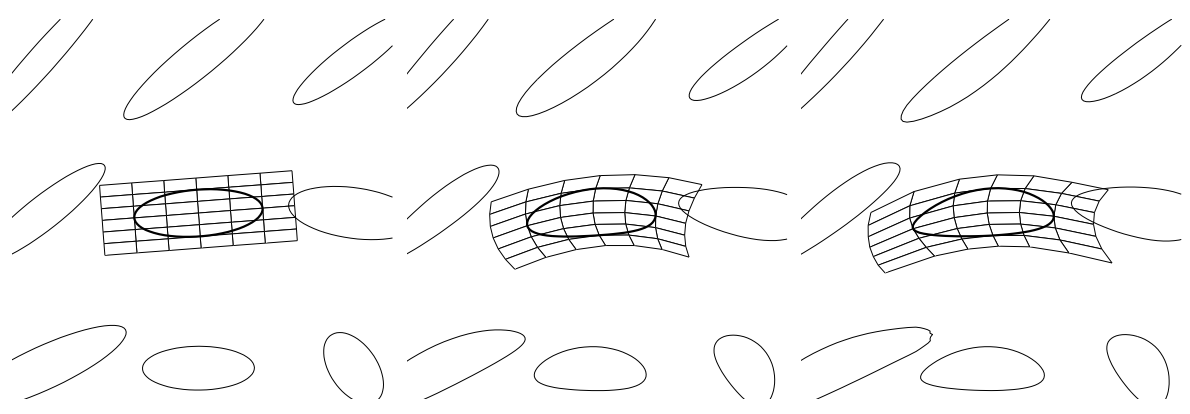

Fig. 5. Left: In a first order approximation of the geodesic normal coordinates, the unit sphere is equivalent to the well known metric ellipsoid. Middle: In a second order approximation of geodesic normal coordinates, similar to exact geodesic coordinates, the unit sphere might be bent. Right: Numerically solving the Geodesic ODE results in almost exact geodesic coordinates. Despite the visible deformation of the Riemannian normal coordinate system attached to the center point, the geodesic sphere glyph looks almost the same in all three examples. For this reason, geodesic spheres may not always be the best choice from a human perceptual point of view. The box glyph overcomes some of these limitations.
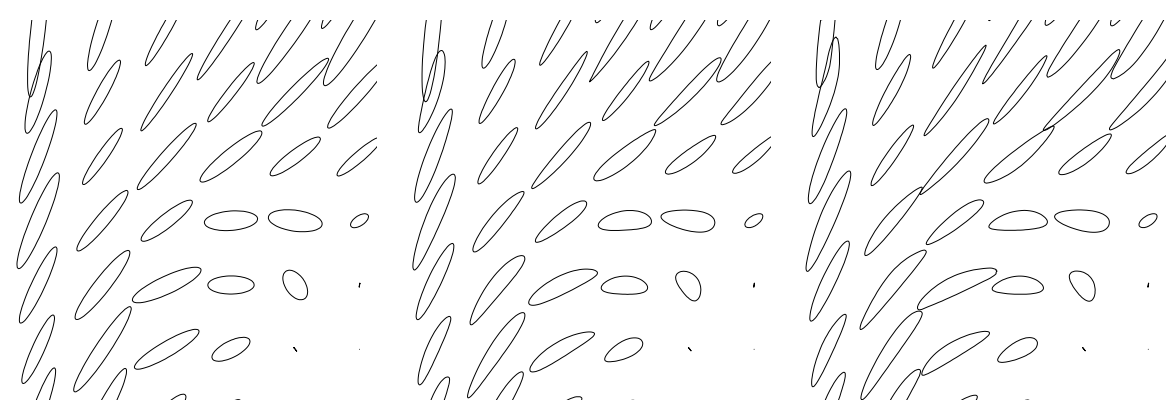

Fig. 6. Left: Metric sphere glyphs painted in a first order approximation of the geodesic normal coordinate system, equivalent to metric ellipsoids. Middle: Metric sphere glyphs painted in a second order approximation of geodesic normal coordinates. Right: Metric sphere glyphs painted in true geodesic coordinates found by numerically solving the geodesic equation.

Fig. 8, we tried glyph-warping on somewhat more exotic glyphs. In the image to the left we have used texture maps of soda cans as tensor glyphs. In the next image we used a creation inspired by superquadratics. Finally in the third image we have used glyph-warping on anisotropy adaptive superquadratics as defined in [13], where isotropic glyphs have been assigned round glyphs and anisotropic glyphs have a more box-shaped appearance. Finally the method was tested on real DTI data, acquired at CMIV. 

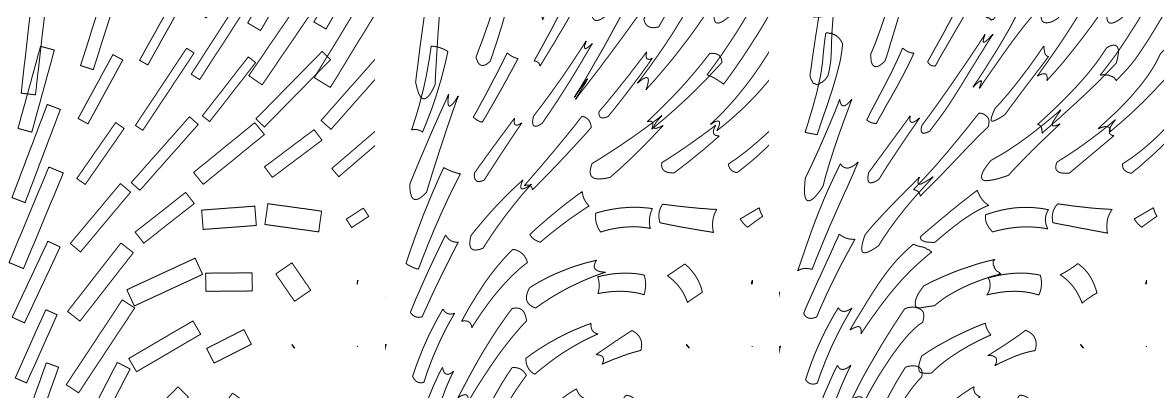

Fig. 7. Left: Tensor box glyphs painted using a first order approximation of geodesic normal coordinates. Middle: Tensor box glyphs painted using a second order approximation of the box glyph. Note that glyphs are not only bent, they also vary in thickness that gives information that is difficult to see when painting geodesic spheres. Right: True geodesic coordinates.

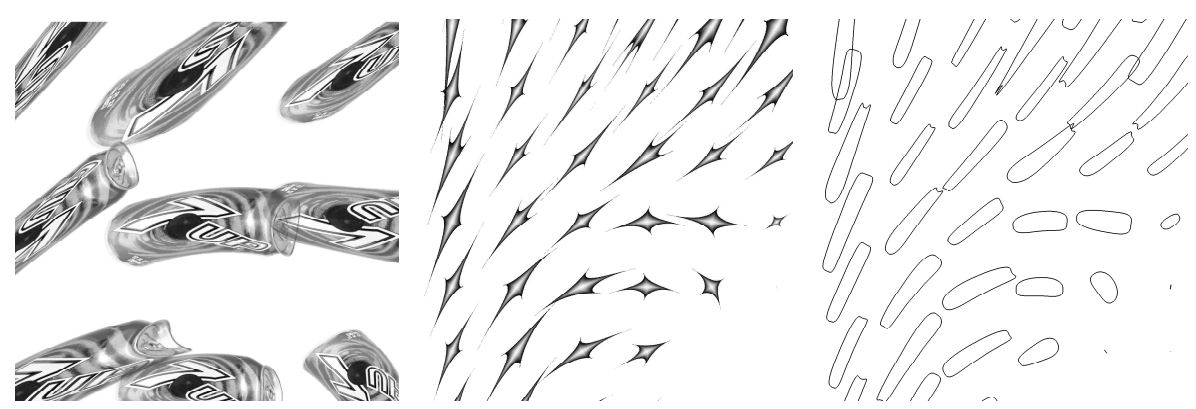

Fig. 8. Warping various glyphs. Left: A soda can glyph. Middle: A superquadratic glyph. Right: Anisotropy adaptive superquadratic glyphs.

\begin{tabular}{c|c|c|} 
& Taylor-1 vs. ODE & Taylor-2 vs. ODE \\
\hline High-resolution & 0.0694 & 0.0475 \\
\hline Low-resolution & 0.1988 & 0.1738 \\
\hline
\end{tabular}

Table 1. A table showing an increased accuracy of the second order approximation to the geodesic coordinate system, compared to the first order approximation. The error measured is the standard deviation of the $\mathrm{x}$ - and $\mathrm{y}$-coordinates for all glyphs in the images shown in Fig. 10, compared to the true ODE solution.

\section{Conclusion}

We have presented a framework for visualization of metric tensor fields in manifolds based on the Riemannian exponential map and its inverse the Riemannian logarithm map . It extends some of the previous methods for painting glyphs based on tensor eigen decomposition and metric spheres. 


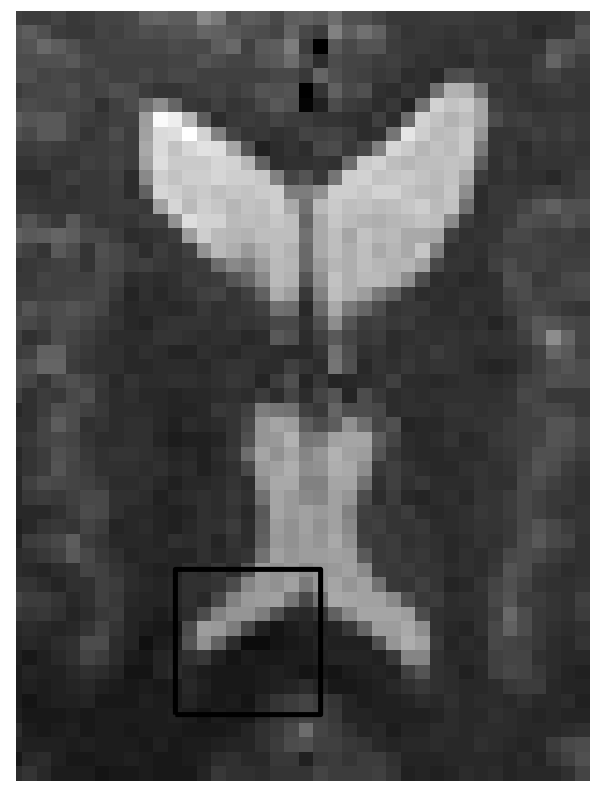

Fig. 9. An anatomical image of a human brain. The square marks the area studied in detail in the DTI examples presented below.

Different from other proposed visualizations of tensor fields using glyphs, this glyph is not strictly a local function of the tensor field in a point, but rather the result from an integration around this point in the manifold. The proposed method for warping glyphs works not only in $\mathbb{R}^{2}$, seen in the experiments, but also easily generalize to $\mathbb{R}^{3}$. By changing the glyph or modifying the tensor field, e.g. by exponentiation of the tensors, we obtain visualizations emphasizing different characteristics in the tensor field. We have derived this glyph warping from derivatives of the metric tensor field, without any reference to any embedding of the manifold (tensor field) being studied. Depending on the need for accuracy or speed, one may choose either numerically accurate geodesic warping by solving the ODE using e.g. the Runge-Kutta method or alternatively, choose the faster version where the bending of the glyphs is calculated using a Taylor approximation of the geodesic.

In summary the Riemannian exponential map, and its inverse the Logarithm map, provides a framework for warping glyphs and visualizing geodesics on a manifolds known only by a space-variant metric in $\mathbb{R}^{n}$.

\section{Acknowledgments}

We thank Magnus Herberthson for valuable discussions on tensors and manifolds and Carl-Fredrik Westin for discussions on the application of these glyphs to Diffusion Tensor MRI data. We are also grateful for the financial support from: The Manifold Valued Signal Processing project, Swedish Research Council (Vetenskapsrådet, grant 2004-4721), CMIV (http://www.cmiv.liu.se), the Center for Medical Image Science 

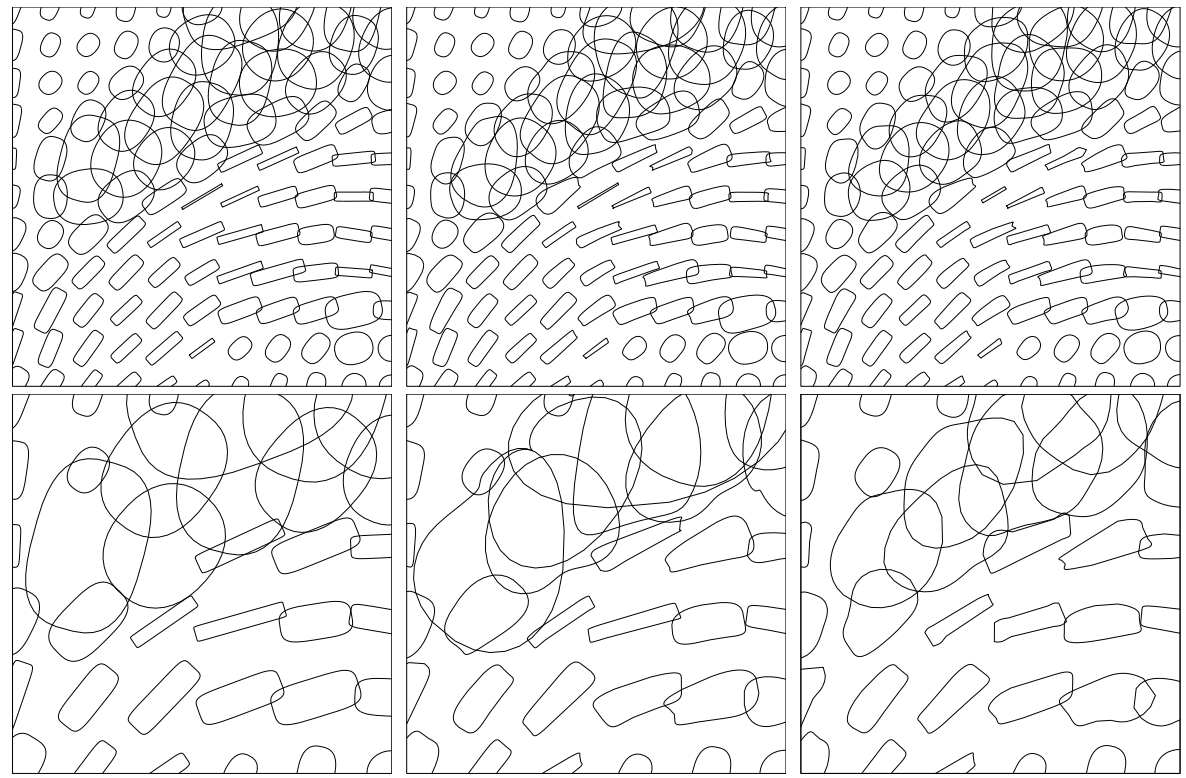

Fig. 10. Tensor glyphs of the planar components of the Diffusion Tensor field in a human brain, shown in high resolution (Top) and low resolution (Bottom). Left: First order glyph approximation. Middle: Second order glyph approximation. Right: exact geodesic coordinates. Apparently the second order approximation introduce errors, compared to the true geodesic coordinates, for large glyphs.

and Visualization and MOVIII (http://www.moviii.isy.liu.se/), the center for Modelling, Visualization and Information Integration funded by the Swedish Foundation for Strategic Research, SSF.

\section{References}

1. V. Arsigny, P. Fillard, X. Pennec, and N. Ayache. Log-Euclidean metrics for fast and simple calculus on diffusion tensors. Magnetic Resonance in Medicine, 56(2):411-421, August 2006.

2. M. Bernstein, V. de Silva, J. Langford, and J. Tenenbaum. Graph approximations to geodesics on embedded manifolds. Technical report, Department of Psychology, Stanford University, 2000.

3. A. Brun. Manifolds in Image Science and Visualization. PhD thesis, Linkping University, 2007. Linkping Studies in Science and Technology Dissertations, No. 1157, ISBN 978-91-85715-02-2.

4. A. Brun, C.-F. Westin, M. Herberthson, and H. Knutsson. Fast manifold learning based on riemannian normal coordinates. In Proceedings of the 14th Scandinavian Conference on Image Analysis (SCIA'05), Joensuu, Finland, June 2005.

5. B. Cabral and L. Casey Leedom. Imaging Vector Fields Using Line Integral Convolution. In J. T. Kajiya, editor, SIGGRAPH93, CGPACS, pages 263-270, New York, 1993. ACM Press/ACM SIGGRAPH. 
6. T. Delmarcelle and L. Hesselink. Visualizing second-order tensor fields with hyper streamlines. IEEE Computer Graphics and Applications, 13(4):25-33, 1993.

7. L. Feng, I. Hotz, B. Hamann, and K. Joy. Anisotropic noise samples. Transactions on Visualization and Computer Graphics, 2008.

8. P. T. Fletcher, C. Lu, S. M. Pizer, and S. Joshi. Principal geodesic analysis for the study of nonlinear statistics of shape. IEEE Transactions on Medical Imaging, 23(8):995-1005, August 2004.

9. G. H. Granlund and H. Knutsson. Signal Processing for Computer Vision. Kluwer Academic Publishers, 1995. ISBN 0-7923-9530-1.

10. I. Hotz, L. Feng, H. Hagen, B. Hamann, K. Joy, and B. Jeremic. Physically based methods for tensor field visualization. In Proceedings of IEEE Visualization 2004, pages 123-130, 2004.

11. C. J. Isham. Modern Differential Geometry for Physicists (World Scientific Lecture Notes in Physics). World Scientific Publishing Company, 1989.

12. H. Karcher. Riemannian center of mass and millifier smoothing. Commun. Pure Appl. Math, 30(5):509-541, 1977.

13. G. Kindlmann. Superquadric tensor glyphs. In Proceedings of IEEE TVCG/EG Symposium on Visualization 2004, pages 147-154, May 2004.

14. G. Kindlmann, D. Weinstein, and D. Hart. Strategies for direct volume rendering of diffusion tensor fields. IEEE Transactions on Visualization and Computer Graphics, 6(2):124-138, 2000.

15. G. Kindlmann and C.-F. Westin. Diffusion tensor visualization with glyph packing. IEEE Transactions on Visualization and Computer Graphics, 12(5), 2006.

16. H. Knutsson, R. Wilson, and G. H. Granlund. Anisotropic non-stationary image estimation and its applications - Part I: Restoration of noisy images. IEEE Transactions on Communications, 31(3):388-397, March 1983.

17. T. McGraw, Baba C. Vemuri, Z. Wang, Yun Chen, M. Rao, and T. Mareci. Line integral convolution for visualization of fiber tract maps from dti. In MICCAI '02: Proceedings of the 5th International Conference on Medical Image Computing and Computer-Assisted Intervention-Part II, pages 615-622, London, UK, 2002. Springer-Verlag.

18. L. O'Donnell, S. Haker, and C.-F. Westin. New approaches to estimation of white matter connectivity in diffusion tensor MRI: Elliptic pdes and geodesics in a tensor-warped space. In Fifth International Conference on Medical Image Computing and Computer-Assisted Intervention (MICCAI'02), pages 459-466, Tokyo, Japan, 2002.

19. Xavier Pennec. Probabilities and statistics on riemannian manifolds: A geometric approach. Research Report 5093, INRIA, January 2004. An extended version will appear in the Int. Journal of Mathematical Imaging and Vision.

20. R. Schmidt, C. Grimm, and B. Wyvill. Interactive decal compositing with discrete exponential maps. In SIGGRAPH '06: ACM SIGGRAPH 2006 Papers, pages 605-613, New York, NY, USA, 2006. ACM.

21. J. A. Sethian. Level Set Methods and Fast Marching Methods. Cambridge, 2001. ISBN 0521642043.

22. A. Sigfridsson, T. Ebbers, E. Heiberg, and L. Wigström. Tensor field visualization using adaptive filtering of noise fields combined with glyph rendering. In Proceedings of IEEE Visualization 2002, pages 371-378, Boston, Massachusetts, 2002. 
23. John N. Tsitsiklis. Efficient algorithms for globally optimal trajectories. IEEE Trans. Automat. Control, 40(9):1528-1538, 1995.

24. J. van Wijk. Spot noise: Texture synthesis for data visualization. In Proceedings of ACM SIGGRAPH 1991, volume 25, pages 309-318. Addison Wesley, 1991.

25. A. Vilanova, Song Zhang, G. Kindlmann, and David H. Laidlaw. An introduction to visualization of diffusion tensor imaging and its applications. In Visualization and Image Processing of Tensor Fields. Springer-Verlag, 2005. In Press.

26. Robert M. Wald. General Relativity. University Of Chicago Press, June 1984.

27. C-F. Westin. A Tensor Framework for Multidimensional Signal Processing. PhD thesis, Linköping University, Sweden, SE-581 83 Linköping, Sweden, 1994. Dissertation No 348, ISBN 91-7871-421-4.

28. L. Ying and E. J Candès. Fast geodesics computation with the phase flow method. ??, 2006.

29. X. Zheng and A. Pang. Hyperlic. In Proceedings of IEEE Visualization 20003, pages 249-256, Seattle, Washington, 2003. 